\title{
ABS: Adaptive Buffer Sizing for Heterogeneous Networks
}

\author{
Yueping Zhang \\ NEC Laboratories America, Inc. \\ Princeton, NJ 08540 \\ Email: yueping@nec-labs.com
}

\author{
Dmitri Loguinov* \\ Texas A\&M University \\ College Station, TX 77843 \\ Email: dmitri@cs.tamu.edu
}

\begin{abstract}
Most existing criteria [3], [5], [9] for sizing router buffers rely on explicit formulation of the relationship between buffer size and characteristics of Internet traffic. However, this is a non-trivial, if not impossible, task given that the number of flows, their individual RTTs, and congestion control methods, as well as flow responsiveness, are unknown. In this paper, we undertake a completely different approach that uses controltheoretic buffer-size tuning in response to traffic dynamics. Motivated by the monotonic relationship between buffer size and loss rate and utilization, we design a mechanism called Adaptive Buffer Sizing (ABS), which is composed of two Integral controllers for dynamic buffer adjustment and two gradient-based components for intelligent parameter training. We demonstrate via ns2 simulations that ABS successfully stabilizes the buffer size at its minimum value under given constraints, scales to a wide spectrum of flow populations and link capacities, exhibits fast convergence rate and stable dynamics in various network settings, and is robust to load changes and generic Internet traffic (including FTP, HTTP, and non-TCP flows). All of these demonstrate that ABS is a promising mechanism for tomorrow's router infrastructure and may be of significant interest for the ongoing collaborative research and development efforts (e.g., GENI and FIND) in reinventing the Internet.
\end{abstract}

\section{INTRODUCTION}

One of the key components of Internet routers is the $\mathrm{I} / \mathrm{O}$ buffer, which is closely linked to various critical performance metrics, including packet loss rate, end-to-end delay, and utilization level. On one hand, router buffers should be large enough to accommodate transient bursts in packet arrivals and hold enough packets to maintain high link utilization. On the other hand, large buffers in turn leads to increased queuing delays and may potentially cause instability of TCP in certain scenarios [27]. Clearly, optimally determining the required buffer size is of immense importance for router manufactures when configuring their routers for the future high-speed Internet and significantly affects the ability of large Internet service providers (ISP) to deliver and guarantee competitive Service Level Agreements (SLA) [33].

As today's Internet rapidly grows in scale and capacity, it becomes widely recognized that the classic bandwidthdelay-product (BDP) [34] rule for sizing router buffers is no longer suitable for the future Internet. In addition, the Internet is foreseeing a disruptive evolution driven by focused

* Supported by NSF grants CCR-0306246, ANI-0312461, CNS-0434940, CNS-0519442, and CNS-0720571. collaborative efforts such as the NSF Global Environment of Network Innovations (GENI) and Future Internet Network Design (FIND) initiatives. This imposes significant challenges as well as great opportunities for all most every corner of Internet technologies, including the next-generation infrastructure for router buffer management. As a consequence, there has emerged in the research community a surge of renewed interest [3], [5], [6], [9], [10], [16], [18], [19], [23], [28], [29], [34], [36], [37] in the buffer-sizing problem during the last five years. However, these results present vastly different, even contradictory, views on how to optimally dimension the buffer of a router interface. In addition, all these results rely on certain assumptions of the incoming Internet traffic and may have limited applications to and exhibit undesirable behavior in other traffic models. In contrast, Kellett et al. [23] take a completely different approach and models the buffer-sizing problem as the Lur'e problem. Under this model, they proposed a dynamic buffer sizing algorithm called Adaptive Drop Tail (ADT). However, the control parameter $K$ depends on the underlying Lur'e formulation and can hardly be obtained without off-line calculation. Thus, it still remains open to develop a model-independent buffer-sizing mechanism that is able to ideally allocate buffers under different traffic patterns.

In this paper, we achieve the goal of buffer sizing by proposing a new buffer management infrastructure, where the router adapts its buffer size to the dynamically changing incoming traffic based on one or more performance constraints. We first formulate buffer sizing as the following problem. Let $B$ be the total size of router's memory and $b_{l}(t)$ be the amount of buffer allocated to link $l$ at time $t$. Then, the problem becomes determining the optimal buffer size for each link $l$ under the constraint that $\sum_{l} b_{l}(t) \leq B$. We then propose that this problem can be alternatively solved by leveraging the monotonic relationship between buffer size $b_{l}$ and various performance metrics (e.g., utilization $u$, loss rate $p$, and queuing delay $q$ ). Rigorously proving this relationship is very difficult and out of scope of the paper. Instead, we provide an intuitive explanation of this result using a simple yet generic congestion control model.

Utilizing this result, we design a buffer management mechanism, called Adaptive Buffer Sizing (ABS), which dynamically determines the minimum buffer size satisfying the target performance constraints based on real-time traffic measurements. 
ABS consists of two sub-controllers $\mathrm{ABS}_{u}$ and $\mathrm{ABS}_{p}$, each of which employs an Integral controller that adapts to dynamics of input traffic by regulating the buffer size based on the error between the measured and target values of utilization and loss rate, respectively. However, we observe that the naive Integral controller $\mathrm{ABS}_{u}$ drives buffers of non-bottleneck routers to infinity. We successfully address this problem by introduce a damping component, such that the resulting $\mathrm{ABS}_{u}$ quickly converges buffers to their equilibrium values in both bottleneck and non-bottleneck routers.

Another challenge is how to tune integral gains for optimal performance. Improper parameter settings may lead to undesirable system behavior, such as slow convergence and persistent oscillations. We solve this problem by associating with each sub-controller a gradient-based parameter training component, which is capable of automatically adapting parameters to achieve their optimal values under the current ingress traffic. We evaluate the resulting controller in ns2 simulations and demonstrate that $\mathrm{ABS}$ is able to deal with generic Internet traffic consisting of HTTP sessions, different TCP variants, and non-TCP flows, is robust to changing system dynamics, and is scalable to link capacities and flow populations, all of which make the concept of ABS an appealing and practical buffer sizing framework for future Internet routers.

The rest of the paper is organized as follows. In Section II, we explore the monotonic relationship between $b, p$, and $u$. In Section III, we present ABS. In Section IV, we examine ABS in a variety of scenarios via ns 2 simulations. In Section V, we review related work on buffer sizing. In Section VI, we conclude our work and point out directions for future work.

\section{Motivation}

While a comprehensive modeling of Internet traffic and its relationship with buffer size $b$ remains open, we show in this subsection that there are strong indications that there exists a monotonic relationship between $b$ and two key performance metrics, loss rate $p$ and utilization $u$. Due to the extreme difficulty of the problem, we do not seek to present a rigorous proof of this monotonic relationship, but provide an intuitive explanation experimentally via $\mathrm{ns} 2$ simulations and analytically using a simple congestion control model. This monotonic relationship serves as motivation and foundation of our adaptive buffer sizing scheme proposed in the following section.

\section{A. Simulation Illustration}

We first empirically examine the impact of the buffer size on the performance of different congestion control protocols using ns 2 simulations. To accomplish this goal, we utilize the framework developed in [35], which incorporates into ns2 the Linux-2.6.16.3 implementations of several proposed TCP variants, including newReno [12], BIC [38], CUBIC [30], HSTCP [11], HTCP [26], STCP [24], Westwood [17], and TCP-LP [25]. The simulation setup is composed of one bottleneck link of capacity $100 \mathrm{mb} / \mathrm{s}$ and ten sources with packet size 1500 bytes and RTTs uniformly distributed in

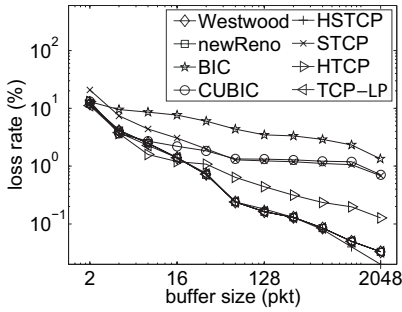

(a) loss rate

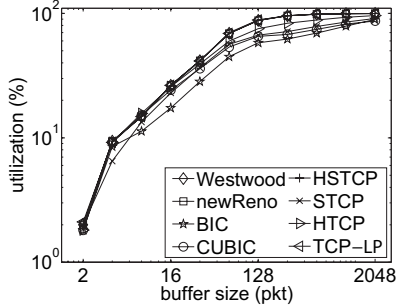

(b) utilization
Fig. 1. Effect of buffer size $b$ on loss rate $p$ and utilization $u$ of several TCP variants.

$[30,50] \mathrm{ms}$. We set buffers of access links to be 2500 packets and verify that no packet is lost at these links in all simulations. The plots of loss rate $p$ and utilization $u$ under different bottleneck buffer sizes are given in Figure 1, from which we can see that $p$ and $u$ respectively monotonically decreases and increases as $b$ grows. Note that the loss curves of CUBIC and STCP in Figure 1(a) appear to be flat when $b$ is between 64 and 1024 packets; however, we verified numerically that they are actually monotonically decreasing.

\section{B. Intuition}

This monotonic relationship is not surprising. It is intuitive clear that a larger buffer can absorb more bursts in packet arrivals, thereby reducing the frequency of packet drops. In addition, assuming the buffer is always depleted between events of packet drops, it takes longer time for sources to saturate a larger buffer so that to experience the next packet loss. Thus, the average loss rate is a decreasing function of the buffer size. At the same time, a larger buffer can hold more packets and thus maintain the bottleneck link at full utilization for a longer time when senders back off in response to congestion. Therefore, the average utilization level proportionally scales with the buffer size. However, the above reasonings assume a depleted queue after each packet loss and may not be obvious otherwise. Thus, it is desirable if we can obtain a more generic explanation with less restrictive assumptions. We next seek to achieve this goal with a simple congestion-control model.

\section{Simple Model}

We note that the goal of this section is not to present a comprehensive congestion control model that is able to formulate generic Internet traffic, but to intuitively explain results observed in the previous section using a simple, but illustrative, model. We start with the definition of $p$ and $u$ in mathematical terms. Consider a scenario where traffic passes through a single-channel FIFO queue of capacity $b$ and service rate $C$. Let $L(t)$ and $A(t)$ respectively denote the number of lost and admitted packets by time $t$. Then, $p$ is defined as the long-term average loss rate $p=\lim _{t \rightarrow \infty} L(t) /(L(t)+A(t))$, $u=\lim _{t \rightarrow \infty} A(t) / C t$ as the average utilization, and $\lambda=$ $\lim _{t \rightarrow \infty} A(t) / t$ as the average input rate.

Using these definitions, we next examine the effect of buffer size on traffic that reacts to congestion using a simple model. 
Denoting by $W_{i}(n)$ the congestion window size of flow $i$ during the $n$-th RTT, we can model a generic congestion control algorithm as following ${ }^{1}$ :

$$
W_{i}(n)=\left\{\begin{array}{ll}
W_{i}(n-1)+\alpha_{i}\left(W_{i}(n-1)\right) & \text { no loss } \\
W_{i}(n-1)-\beta_{i}\left(W_{i}(n-1)\right) & \text { loss }
\end{array},\right.
$$

where $\alpha_{i}(\cdot)$ and $\beta_{i}(\cdot)$ are non-negative functions and each discrete time step corresponds to one RTT. This simple model subsumes a wide spectrum of loss-based congestion control protocols, including AIMD (e.g., Reno [1] and Westwood [17]), MIMD (e.g., Scalable TCP [24]), and many other existing TCP variants (e.g., BIC [38], TCP-LP [25], and HSTCP [11]). Note that delay-based schemes (e.g., FAST [22] and Vegas [7]) generally are not sensitive to buffer size $b$ as long as it is kept larger than the stationary queue length $q^{*}$ of the system. However, when $b<q^{*}$ these methods experience packet losses and their responses can also be modeled by (1). Moreover, since (1) allows different response functions $\alpha_{i}(\cdot)$ and $\beta_{i}(\cdot)$ for different flows $i$, this model applies to scenarios where the traffic is generated by a mixture of protocols.

Assuming $N$ sources with homogeneous RTTs access a single link of capacity $C$ and letting $q(n)$ be the queue length at time $n$, we can model the queuing dynamics as:

$$
q(n)=\min \left((q(n-1)+X(n)-C)^{+}, b\right),
$$

where $b$ is the buffer size and $X(n)=\sum_{i=1}^{N} W_{i}(n)$ is the total number of arrivals during the $n$-th RTT. Assuming that $W_{i}(n)$ of each source $i$ is bounded above by $W_{\max }$, the system dynamics can be represented by a discrete Markov chain with state $S_{n}=\left[W_{1}(n), W_{2}(n), \ldots, W_{N}(n)\right]$ and state space $O:\left[1,2, \ldots, W_{\max }\right]^{N}$.

Let $Z(n)$ be the number of dropped packets during the $n$-th RTT: $Z(n)=(q(n-1)+X(n)-C-b)^{+}$. Define $v(n)=Z(n) / X(n)$ as the average loss rate during this RTT. Assuming packet loss rate of each flow is independent of each other, we have $\operatorname{Pr}\left\{W_{i}(n+1)=W_{i}(n)+\alpha_{i}\left(W_{i}(n)\right)\right\}=$ $(1-v(n))^{W_{i}(n)}$ and $\operatorname{Pr}\left\{W_{i}(n+1)=W_{i}(n)-\beta_{i}\left(W_{i}(n)\right)\right\}=$ $1-(1-v(n))^{W_{i}(n)}$. Based on this, we can derive the transition probability between any pair of states. Furthermore, it is clear that the transition probability depends only on the previous state, which implies that series $\left\{S_{n}\right\}$ is a Markov chain. Then, the following result is easy to obtain.

Theorem 1: The Markov chain defined by (1)-(2) always converges to a stationarity distribution.

According to Theorem 1, for any fixed $N$ and starting from any initial state, the Markov chain defined by (1)-(2) always converges to its steady state. Thus, we omit the transient phase in the rest of the section and only examine the queuing process under traffic generated by a stationary Markov chain. In such a scenario, the following result shows that packet loss rate $p$ scales inversely proportionally to the buffer size $b$.

\footnotetext{
${ }^{1}$ We assume $W_{i}(n)$ are rounded to integers during calculations and omit the corresponding ceiling function for brevity.
}

Theorem 2: Loss probability $p$ in a finite queue fed by traffic governed by a stationary Markov chain defined by (1)(2) monotonically decreases in queue size $b$.

Proof: Under the stationary Markov chain defined by (1)-(2), denote by $S_{n}$ the state at time $n$, by $M$ the number of states, and by $\pi=\left[\pi_{1}, \pi_{2}, \ldots, \pi_{M}\right]$ the stationary probability vector of each state (i.e., $\pi_{i}=\operatorname{Pr}\left\{S_{n}=i\right\}$ ). Further let $A_{i, j}(k)$ be the probability that the chain goes from state $i$ to $j$ and the next arrival has $k$ packets, i.e., $A_{i, j}(k)=\operatorname{Pr}\left\{X(n+1)=k, S_{n+1}=j \mid S_{n}=i\right\}$. Then, define $\boldsymbol{A}_{k}$ as the probability matrix whose $(i, j)$-th element is $A_{i, j}(k)$. Using these variables, we can represent the traffic density $\rho$ as $\rho=\pi \sum_{k=1}^{\infty} k \boldsymbol{A}_{k} \boldsymbol{e}$, where $\boldsymbol{e}$ is a column vector with all elements equal to one.

We next express loss probability of a finite buffer of size $b$ in terms of the queue length distribution of an infinite buffer, whose queuing process $q^{\prime}(n)$ is given by:

$$
q^{\prime}(n)=\left(q^{\prime}(n-1)+X(n-1)-c\right)^{+} .
$$

Consider the steady-state probability matrix $\Delta$ of the infinite buffer, where the $(k, i)$-th element $\Delta_{k, i}$ is:

$$
\Delta_{k, i}=\lim _{n \rightarrow \infty} \operatorname{Pr}\left\{X(n)=k, S_{n}=i\right\} .
$$

Then, according to [21, Theorem 4], under arrivals governed by the same stationary Markov chain, loss probability $p$ of a finite buffer of size $b$ is represented by:

$$
p=\frac{(1-\rho) \sum_{k=b+1}^{\infty} \Delta_{k} A_{0} e}{\rho \sum_{k=0}^{b} \Delta_{k} A_{0} e},
$$

where $\boldsymbol{\Delta}_{k}$ is the $k$-th row vector of $\boldsymbol{\Delta}$. Since the Markov chain is stationary, all variables in the last equation are constant. Thus, it follows that loss rate $p$ monotonically decreases as buffer size $b$ increases.

Moreover, it is clear that utilization $u$ scales inversely to loss rate $p$ according to a general TCP model of the form $r=c / p^{d}$, where $r$ is the throughput and $c$ and $d$ are constants [38]. This holds for various TCP flavors including Reno, BIC, HSTCP, and STCP. Further notice that according to Theorem 2, loss rate $p$ scales inversely to $b$. This implies that utilization $u$ monotonically increases in $b$. In addition, it is rather obvious that queuing delay $q$ scales proportionally to buffer size $b$. Thus, in the rest of the paper we assume the monotonic relationship between $u$ and $b$ and $q$ and $b$.

Finally, we should emphasize that although system (1) is generic enough to represent the increase/decrease behavior of a wide class of congestion control algorithms, it is by no means comprehensive. To make the model complete, one should additionally consider factors such as heterogeneous delay, slow start, timeouts, and retransmissions. The pure purpose of this model is to provide an intuitive explanation of the monotonic relationship between $b$ and $u, p$. This result motivates us to design a dynamic buffer-sizing mechanism presented below.

\section{AdAPtive BufFER SizIng (ABS)}

In this section, we describe a dynamic buffer sizing framework that is adaptive to dynamics and uncertainties of input 
traffic while maintaining the system under target performance constraints such as loss rate $p^{*}$ and utilization $u^{*}$. As an example of this framework, we start with a simple mechanism, progressively identify and overcome its underlying drawbacks, and eventually arrive at the final controller that we call ABS.

\section{A. General Consideration}

To design a buffer sizing mechanism, first it is necessary to understand how buffers are managed in current commercial routers. The memory system in a Cisco 3600 series router [8], for instance, is composed of the main processor memory, shared (packet) memory, flash memory, nonvolatile random access memory (NVRAM), and erasable programmable read only memory (EPROM). Among them, we are particularly interested in shared (packet) memory, which is used for packet buffering by the router's network interfaces. Specifically, each interface is associated with an input hold buffer (IHB), which resides in the system buffer of shared memory and is used to store packets for transfer between fast switching and process switching code. For each packet arriving into an interface, the interface driver writes it into an IHB. An incoming packet is immediately dropped if the IHB reaches its maximum size, which is static and does not grow or shrink based on demands. Our goal in this paper is redesign IHB such that its size adapts to dynamics of the incoming traffic.

We note that it is important to distinguish the framework of dynamic buffer sizing from the large class of AQM algorithms (e.g., RED [13], REM [4], and PI [20]). These methods operate within a given buffer size $b_{l}$ and aim to stabilize the queue occupancy (or queuing delay) at a certain target level, which is a portion of the selected buffer size $b_{l}$. Thus, AQM is unable to solve issues associated with incorrectly sized router buffers.

To better see this, we test several TCP variants under REM using ns2 simulations. Recall that an REM-enabled router dynamically updates its packet dropping/marking probability by monitoring the discrepancy between the aggregate input rate $y(t)$ and link capacity $C$ and the difference between the current queue length $q(t)$ and its target value $q^{*}$. In the steady state, the system achieves $y(t)=C$ and $q(t)=q^{*}$. In our simulations, we use a simple "dumbbell" topology with a single REM $\left(q^{*}=50\right.$ pkts $)$ link of capacity $C=10 \mathrm{~ms} / \mathrm{b}$ shared by 20 TCP sessions. We use marking at the REM router and enable ECN at end-users. As seen from Table I, if we set buffer size of the bottleneck link to $b=100$ pkts, which is greater than REM's target queue size $q^{*}=50$ pkts, REM successfully maintain the queue size close to $q^{*}$ while achieving $100 \%$ utilization and $0 \%$ packet loss for all TCP variants. However, if we set buffer size $b$ below $q^{*}$, the bottleneck link suffers significant under-utilization and prohibitively high loss rate.

In contrast, dynamic buffer-sizing mechanisms focus on determining the optimal size of the physical buffer. This way, the router can efficiently allocate its available buffers among different memory-sharing interfaces, hereby achieving preagreed QoS requirements, shrinking the required space of the main memory, and reducing the system cost and board space.
TABLE I

PERFORMANCE OF DIFFERENT TCP VARIANTS WITH REM $\left(q^{*}=50\right.$ PKTS $)$ UNDER DIFFERENT BUFFER SIZES.

\begin{tabular}{c|ccc|ccc}
\hline & \multicolumn{3}{|c|}{$b=100 \mathrm{pkts}$} & \multicolumn{3}{c}{$b=10 \mathrm{pkts}$} \\
& $q(\mathrm{pkts})$ & $p(\%)$ & $u(\%)$ & $q(\mathrm{pkts})$ & $p(\%)$ & $u(\%)$ \\
\hline Reno & 56.14 & 0.00 & 100.00 & 5.41 & 9.88 & 84.78 \\
BIC & 52.88 & 0.00 & 100.00 & 5.27 & 9.04 & 86.52 \\
CUBIC & 52.48 & 0.00 & 100.00 & 4.92 & 7.84 & 87.22 \\
HSTCP & 56.73 & 0.00 & 100.00 & 4.96 & 9.60 & 86.59 \\
STCP & 54.38 & 0.00 & 100.00 & 5.40 & 12.48 & 83.74 \\
HTCP & 57.77 & 0.00 & 100.00 & 4.60 & 8.20 & 87.46 \\
Westwood & 54.61 & 0.00 & 100.00 & 4.99 & 10.08 & 84.50 \\
\hline
\end{tabular}

Dynamic buffer sizing schemes can overcome the problem of improper buffer sizing that $\mathrm{AQM}$ is unable to solve or may be combined with AQM methods to achieve desired performance. In existing Internet routers where memory is already fixed, the proposed approach is also valuable since it guarantees the minimum queuing delay in each interface under predetermined performance constraints. Additionally, ABS lends ISPs and router manufactures great freedom in choosing preferred constraints when configuring their routers.

\section{B. Controller Design}

Although the underlying differential/difference equations describing the effect of router buffer size on Internet traffic remain unknown, it follows from the last section an important property of this relationship - monotonicity. This implies that for any given feasible loss rate $p^{*}$ (or utilization $u^{*}$ ) under stationary input traffic, there exists a unique buffer size $b^{*}$ such that the resulting system achieves $p^{*}$ (or $u^{*}$ ). In addition, this monotonic relationship gives us a hint of the correct direction in which we should adjust the buffer size. Specifically, assuming target loss rate $p^{*}$ and its actual value $p(n)$ measured at time $n$, the router buffer size $b(n)$ should increase if $p(n)>p^{*}$ and decrease otherwise. Analogously, given $u^{*}$ and $u(n), b(n)$ should decrease if $u(n)>u^{*}$ and increase otherwise. This result allows us to develop simple yet robust controllers to adaptively size router buffers to satisfy given system constraints.

One natural candidate for achieving this goal is the Integral controller. First, consider the controller under the loss rate constraint, in which case $b_{p}(n)$ denotes the buffer size at time $n$ and $e_{p}(n)=p(n)-p^{*}$. Then, the time-domain Integral controller can be represented by the following difference equation:

$$
b_{p}(n)=b_{p}(n-1)+I_{p} T\left(p(n)-p^{*}\right),
$$

where $T$ is the sampling interval and $I_{p}$ is the integral gain. Similarly, we obtain the control equation of $b_{u}(n)$ under the utilization constraint: ${ }^{2}$

$$
b_{u}(n)=b_{u}(n-1)-I_{u} T\left(u(n)-u^{*}\right),
$$

where $I_{u}$ is the integral gain. It is noteworthy to point out that since $p(n)$ monotonically decreases with $b(n)$ while $u(n)$

\footnotetext{
${ }^{2}$ Additional constraints, such as queuing delay, can be easily adopted in our method. For ease of presentation, we only concentrate on loss rate and link utilization in the paper.
} 
(a) $u^{*}=95 \%, p^{*}=0.5 \%$

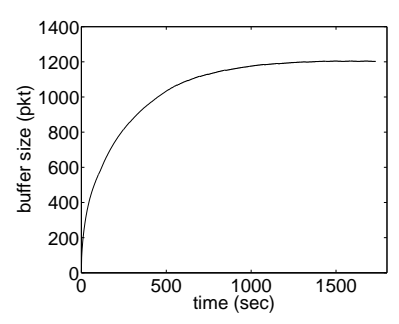

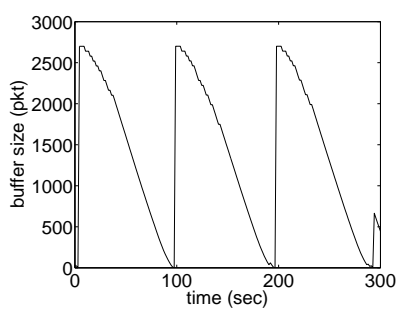

(b) $u^{*}=70 \%, p^{*}=5 \%$
Fig. 2. $\operatorname{ABS}\left(I_{u}=I_{p}=3000\right.$ and $\left.T=200 \mathrm{~ms}\right)$ without parameter training in a network with a single link of capacity $10 \mathrm{mb} / \mathrm{s}$ and 20 TCP flows.

increases with $b(n)$, controllers (6) and (8) have opposite signs before their respective integral gain.

However, the last controller invites a serious problem if deployed in non-bottleneck routers. This is because a nonbottleneck router is always under-utilized regardless of its buffer size. Thus, if $u^{*}$ is set to be above the maximally achievable utilization level of the link, the router always have $u(n)<u^{*}$ and drives its buffer size to infinity. We overcome this problem by modifying (7) as follows:

$$
b_{u}(n)=b_{u}(n-1)-I_{u} T(u(n)-u(n-1))\left(u(n)-u^{*}\right) .
$$

Compared to (7), the extra term $u(n)-u(n-1)$ in (8) is to damp the effect of $\left(u(n)-u^{*}\right)$. Specifically, in the steady state of a non-bottleneck router, we must have $u(n)-u(n-1)=0$, which forces the second term of (8) to converge to zero and prevents $b_{u}(n)$ from diverging to infinity.

Now, we have two buffer sizes $b_{u}(n)$ and $b_{p}(n)$ based on the utilization and loss rate constraints, respectively. Similar to BSCL [9] , the minimum buffer size $b(n)$ satisfying both requirements should be the larger of $b_{p}(n)$ and $b_{u}(n)$, i.e.,

$$
b(n)=\max \left(b_{u}(n), b_{p}(n)\right) .
$$

We call the hybrid controller (6)-(9) Adaptive Buffer Sizing (ABS) scheme and sub-controllers (6) and (8) $\mathrm{ABS}_{p}$ and $\mathrm{ABS}_{u}$, respectively. In practice, an ISP can choose to use these two controllers together or separately depending upon the SLA or actual needs. Note that ABS does not rely on comprehensive prior knowledge of the system being controlled, but adapts the controller according to errors of the output signals $u(n)$ and $p(n)$. As a consequence, $\mathrm{ABS}$ is expected to work in practical network settings and be robust to real Internet traffic (more on this below).

This controller works very well in many cases. However, its main limitation lies in the difficulty in choosing the optimal gain parameters $I_{u}$ and $I_{p}$. Specifically, if they are chosen too small, the system may suffer from a sluggish convergence rate to the equilibrium; however, if they are set too large, the system may exhibit exceedingly aggressive adaptation behavior and persistently oscillate around, instead of converging to, the stationary point. This phenomenon is illustrated in Figure 2, where 20 TCP flows share an ABS-equipped bottleneck link of capacity $10 \mathrm{mb} / \mathrm{s}$. We set integral gains $I_{p}=I_{u}=3000$ and control interval $T=200 \mathrm{~ms}$. As seen in the figures, ABS is stable and converges the buffer size to 1200 packets when $u^{*}=95 \%$ and $p^{*}=0.5 \%$. However, when $u^{*}=70 \%$ and $p^{*}=5 \%$ the system becomes unstable and the buffer size periodically oscillates between 1 and 2700 packets.

Due to the lack of the knowledge of the differential equations describing the system, it is unlikely that any off-line pre-training of the controller's parameters $I_{u}$ and $I_{p}$ can be effective. Even if such a method could exist, parameters trained for a particular system setting may immediately become inappropriate as the traffic dynamics evolve. We next seek to overcome this issue by designing a parameters tuning mechanism that is able to adaptively converge the control parameters to their optimal values for the current system configuration.

\section{Adaptive Parameters Training}

It is clearly a non-trivial task to find the optimal parameters for controlling such a complex system as the Internet, which is especially the case provided that the system has an unknown underlying model and dynamically changes over time. However, we manage to achieve this goal using a simple scheme, which combines the output error [2] method and the gradient descent [32] technique. In what follows, we explain our method in the context of $\mathrm{ABS}_{u}$ and note that the mechanism for $\mathrm{ABS}_{p}$ can be obtained similarly.

Denote by $I_{u}(n)$ the instantaneous value of integral gain $I_{u}$ at time $n$. Then, rewrite $\mathrm{ABS}_{u}$ 's control equation (8) as:

$$
b_{u}(n)=f_{u}\left(u^{*}, b_{u}(n-1), u(n), I_{u}(n)\right),
$$

where function $f_{u}($.$) is given by the right-hand side of (8).$ Suppose that the router, at the end of the $n$-th control interval, sets its buffer size to $b_{u}(n)$ based on (10) and observes that link utilization becomes $u(n+1)$ during the next interval. Then, we know that if we set $u^{*}=u(n+1)$ as the target utilization, $b_{u}(n)$ must be the optimal output of controller (10) under the same traffic pattern and given buffer size $b_{u}(n-1)$ and utilization $u(n)$. This is equivalent to saying that under the optimal control gain $I_{u}^{*}$, we must have the following equation:

$$
b_{u}(n)=f_{u}\left(u(n+1), b_{u}(n-1), u(n), I_{u}^{*}\right) .
$$

Thus, at every control step, we get the exact value of the inverse function of the controlled plant [2]. Hence, it remains to adaptively adjust $I_{u}(n)$ to achieve its optimal value $I_{u}^{*}$, which translates into the following optimization problem.

First, define

$$
b_{u}^{\prime}(n)=f_{u}\left(u(n+1), b_{u}(n-1), u(n), I_{u}(n)\right)
$$

as the actual controller's output under the current integral gain $I_{u}(n)$. This value is not used to decide the buffer size, but is applied to the following calculation: $F_{u}(n)=b_{u}^{\prime}(n)-b_{u}(n)$, which is the difference between the actual and optimal outputs. Then, the optimal control gain $I_{u}^{*}$ under the current traffic is the one that minimizes $F_{u}(n)$. To find this optimal parameter, we use the gradient-descent algorithm.

According to the gradient-decent method, since function $F_{u}(n)$ is differentiable at $I_{u}(n)$, it decreases fastest along the 


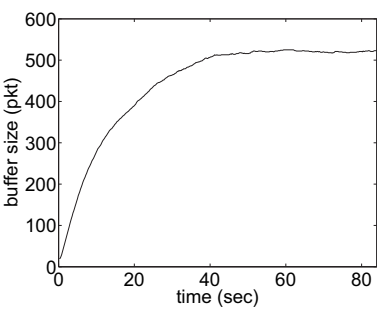

(a) $u^{*}=95 \%, p^{*}=0.5 \%$

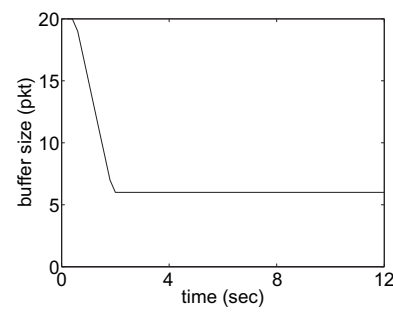

(b) $u^{*}=70 \%, p^{*}=5 \%$
Fig. 3. ABS with parameter training in a network with a single link of capacity $10 \mathrm{mb} / \mathrm{s}$ and $20 \mathrm{TCP}$ flows.

direction of its gradient $\nabla F_{u}\left(I_{u}(n)\right)$, which is the derivative of $F_{u}(n)$ with respect to $I_{u}(n)$. Thus, at every control step, if the router updates parameter $I_{u}(n)$ as follows:

$$
I_{u}(n+1)=I_{u}(n)-\gamma \nabla F_{u}\left(I_{u}(n)\right),
$$

with step size $\gamma$ (which is set to 1 in the paper), then we have that sequence $F_{u}\left(I_{u}(1)\right) \geq F_{u}\left(I_{u}(2)\right) \geq \ldots$, which eventually converges to zero. In this case, $I_{u}(n)$ reaches $I_{u}^{*}$.

Invoking (8), we simply have:

$$
\begin{aligned}
\nabla F_{u}\left(I_{u}(n)\right) & =\frac{d F_{u}(n)}{d I_{u}(n)} \\
& =T(u(n+1)-u(n))\left(u(n+1)-u^{*}\right) .
\end{aligned}
$$

Combining the last two equations, we arrive at the following parameter tuning rule for $I_{u}(n)$ :

$$
I_{u}(n+1)=I_{u}(n)-\gamma T(u(n+1)-u(n))\left(u(n+1)-u^{*}\right) .
$$

Following the above techniques, we can derive the following parameter training rule for $I_{p}(n)$ in $\mathrm{ABS}_{p}$ :

$$
I_{p}(n+1)=I_{p}(n)-\gamma T\left(p^{*}-p(n+1)\right) .
$$

So far, we have finished the design process of ABS, which now consists of two basic Integral controllers (6) and (8) and two parameter training components (15)-(16). Note that the resulting system is independent of the exact model of the controlled plant and highly adaptive to the plant's changing system dynamics. In addition, the proposed parameter training mechanism is not limited to our particular case, but applicable to other systems with multiple parameters to be optimized.

To examine performance of the resulting controller, we rerun simulations in Figure 2. The simulation results are illustrated in Figure 3, from which we can see that in both cases ABS successfully converges the buffer size to its stationary value and exhibits much faster convergence speed compared to its original version shown in Figure 2. We note that since traffic loads at Internet routers change slowly over time, buffer sizing schemes are able to utilize long sampling intervals to filter out noise in measurements and achieve more accurate approximation of the systems average behavior. Thus, the convergence rate of ABS should not be confused with that of a congestion control or AQM protocols, whose control actions are usually performed at the time-scale of milliseconds. However, as we demonstrate later in the paper, ABS is in fact very responsive and works well under highly bursty Internet traffic. Finally, we observe in both simulations that gain parameters $I_{u}$ and $I_{p}$ indeed converge to their respective optimal value.

\section{Performance}

We next demonstrate via ns2 simulations performance of ABS under a wide range of flow populations and link capacities, dynamically changing traffic loads, synthetic web traffic, and mixture of TCP and non-TCP flows.

\section{A. Implementation}

ABS admits a very simple implementation and incurs minimal computational overhead. Specifically, the router maintains two counters $S_{1}$ and $S_{2}$ to respectively record the amount of data enqueued and dropped by the router during the current sampling interval. For each incoming packet $k$ with size $s_{k}$, either $S_{1}$ or $S_{2}$ is incremented by $s_{k}$ depending on whether the packet is admitted. Thus, there is only one addition per packet. At the end of the $n$-th interval, the router computes loss rate using $p(n)=S_{1} /\left(S_{1}+S_{2}\right)$ and utilization using $u(n)=\left(S_{1}+S_{2}\right) /(C T)$ where $C$ is the link's capacity. Then, the router calculates the gain parameters based on (15)-(16) and decides its buffer size according to (6)-(9). Since these operations are performed once every control interval (which is set to 20 seconds in the paper), the incurred overhead is negligible.

In practice, dynamic buffer sizing may encounter some implementation issues. For instance, one such problem is memory fragmentation, which occurs when the router frequently allocates and releases differently sized memory blocks and as a result the memory space contains a lot of small unused pieces. This problem can be mitigated by increasing granularity of memory allocation, i.e., allocating in fixed-size chunks of memory. Sizes of chunks can be 2048 bytes, 4096 bytes, or other values depending on the system. However, for purpose of demonstration, we do not include this mechanism in simulations shown below.

\section{B. Scalability}

In this subsection, we compare performance of existing buffer sizing mechanisms (i.e., BDP, Stanford model, BSCL, and ABS) under different link capacities $C$ and flow populations $N$. Note that due to lack of publicly available implementation and unspecified control parameter $K$, we do not include ADT in this comparison study. We use a "dumbbell" topology with $N$ long-lived TCP flows, whose RTTs are randomly distributed in $[30,30+2 N] \mathrm{ms}$. As suggested in [9], we use the harmonic average RTT $R_{e}$ for the BDP rule. For the Stanford model, we use equation $b=2 R_{e} C / \sqrt{N}$. In BSCL, we set the loss synchronization factor $\alpha$ to 0.6 . In both BSCL and ABS, we set $u^{*}=98 \%$ and $p^{*}=2 \%$.

We first fix link capacity $C=16 \mathrm{mb} / \mathrm{s}$ and vary $N$ between $[2,1024]$. The simulation results are illustrated in Table II, in which data are averaged over the second half of each simulation to eliminate initial transient effects. As shown in 
TABLE II

Performance of Existing Buffer-Sizing Strategies in a Single-Link Network with Different Numbers of Flows $N(C=10$ mb/s, $u^{*}=98 \%$, AND $p^{*}=2 \%$ )

\begin{tabular}{c|ccc|ccc|ccc|ccc}
\hline$N$ & & BDP & & \multicolumn{3}{c|}{ Stanford } & \multicolumn{3}{c}{ BSCL } & \multicolumn{3}{c}{ ABS } \\
& $b(\mathrm{pkts})$ & $p(\%)$ & $u(\%)$ & $b(\mathrm{pkts})$ & $p(\%)$ & $u(\%)$ & $b(\mathrm{pkts})$ & $p(\%)$ & $u(\%)$ & $b(\mathrm{pkts})$ & $p(\%)$ & $u(\%)$ \\
\hline 2 & 110 & 0.08 & 94.3 & 155 & 0.06 & 95.8 & 55 & 0.11 & 89.6 & 287 & 0.03 & 97.9 \\
4 & 116 & 0.18 & 94.3 & 116 & 0.18 & 94.3 & 47 & 0.32 & 85.5 & 232 & 0.09 & 97.8 \\
8 & 128 & 0.42 & 93.5 & 91 & 0.55 & 88.5 & 45 & 0.83 & 78.6 & 229 & 0.23 & 97.9 \\
16 & 150 & 1.05 & 94.9 & 75 & 1.6 & 87.8 & 45 & 1.8 & 83.4 & 227 & 0.74 & 97.9 \\
32 & 190 & 1.76 & 98.3 & 68 & 2.88 & 92.8 & 45 & 3.24 & 89.8 & 151 & 2.04 & 97.9 \\
64 & 261 & 2.76 & 99.9 & 66 & 4.85 & 95.8 & 94 & 4.51 & 97.6 & 443 & 2.00 & 100.0 \\
128 & 383 & 4.06 & 100.0 & 68 & 7.08 & 97.5 & 358 & 4.18 & 100.0 & 941 & 2.05 & 100.0 \\
256 & 595 & 5.11 & 100.0 & 75 & 9.38 & 98.8 & 922 & 4.24 & 100.0 & 2006 & 2.03 & 100.0 \\
512 & 965 & 5.69 & 100.0 & 86 & 11.42 & 99.8 & 2106 & 4.19 & 100.0 & 4210 & 2.00 & 100.0 \\
1024 & 1618 & 6.47 & 100.0 & 102 & 13.45 & 100.0 & 4569 & 3.94 & 100.0 & 8613 & 2.03 & 100.0 \\
\hline
\end{tabular}

TABLE III

Performance of Existing Buffer-Sizing Strategies in a Single-Link Network with Different Link Capacities $C\left(N=16, u^{*}=98 \%\right.$, AND $p^{*}=2 \%$ )

\begin{tabular}{c|ccc|ccc|ccc|ccc}
\hline \multirow{2}{*}{$C(\mathrm{mb} / \mathrm{s})$} & \multicolumn{3}{|c|}{ BDP } & \multicolumn{3}{c|}{ Stanford } & \multicolumn{3}{c|}{ BSCL } & \multicolumn{3}{c}{ ABS } \\
& $b(\mathrm{pkts})$ & $p(\%)$ & $u(\%)$ & $b(\mathrm{pkts})$ & $p(\%)$ & $u(\%)$ & $b(\mathrm{pkts})$ & $p(\%)$ & $u(\%)$ & $b(\mathrm{pkts})$ & $p(\%)$ & $u(\%)$ \\
\hline 2 & 19 & 9.7 & 99.2 & 10 & 12.7 & 97.5 & 76 & 3.7 & 99.9 & 148 & 2.08 & 99.9 \\
4 & 38 & 4.8 & 98.4 & 19 & 6.6 & 95.1 & 53 & 4.0 & 99.1 & 131 & 2.03 & 99.7 \\
8 & 75 & 2.4 & 96.1 & 38 & 3.03 & 91.9 & 17 & 4.3 & 79.5 & 120 & 1.85 & 97.9 \\
16 & 150 & 1.05 & 94.9 & 75 & 1.6 & 87.8 & 45 & 1.8 & 83.4 & 227 & 0.74 & 97.9 \\
32 & 300 & 0.4 & 97.3 & 150 & 0.6 & 91.2 & 100 & 0.8 & 86.2 & 333 & 0.35 & 97.9 \\
64 & 600 & 0.13 & 98.5 & 300 & 0.2 & 94.3 & 211 & 0.3 & 90.9 & 496 & 0.15 & 97.9 \\
128 & 1200 & 0.05 & 99.2 & 600 & 0.08 & 96.4 & 432 & 0.09 & 93.0 & 780 & 0.06 & 97.8 \\
256 & 2400 & 0.021 & 99.4 & 1200 & 0.03 & 98.1 & 875 & 0.04 & 95.2 & 1164 & 0.028 & 97.9 \\
512 & 4799 & 0.003 & 99.6 & 2400 & 0.008 & 98.6 & 1760 & 0.01 & 97.7 & 1759 & 0.012 & 98.1 \\
1024 & 9597 & 0.0003 & 99.6 & 4799 & 0.0007 & 98.2 & 3531 & 0.0009 & 96.6 & 3860 & 0.0008 & 97.9 \\
\hline
\end{tabular}

the table, when the number of flows is small, both the BDP and Stanford rules are not very successful in achieving their design goal (i.e., high link utilization). As $N$ becomes large, both methods do achieve high utilization, but in the expense of high loss rates. This is especially evident in the Stanford model, whose loss rate is $13.45 \%$ when there are 1024 flows. Capability of controlling loss rate is improved in BSCL; however, it still cannot achieve the target loss rate $p^{*}=2 \%$ and suffer from low link utilization when the number of flows is small. In contrast, ABS achieves its design goal under all flow populations. Specifically, when $N \leq 32$ and utilization is the primary constraint, ABS successfully maintains link utilization at close to its target value $u^{*}=98 \%$. As $N$ grows and the loss rate constraint becomes dominant, ABS is still able to effectively allocate buffer such that the average loss rate is within a close neighborhood of $p^{*}=2 \%$.

It is worth noting that as seen from Table II, when $N=$ 1024, ABS converges the buffer size to 8613 packets. This buffer size translates into a queuing delay of 10 seconds, which is prohibitively high for most applications. However, this is not a problem of ABS, but a consequence of an unrealistic choice of $p^{*}$. In practice, router manufactures and ISPs are free to adjust $u^{*}$ and $p^{*}$ according to the type of service they agree to provide and the actual traffic situation. To avoid exceedingly large queuing delay, they can increase the link capacity or enforce a predetermined upper bound of buffer size to prevent queuing delay from growing beyond a certain threshold value.

We next set $N=16$ and examine scalability of these methods to link capacities. As seen from Table III, the BDP and Stanford rules result in significant packet loss under small link capacities $(C \leq 4 \mathrm{mb} / \mathrm{s})$. Although they achieve both low loss rate and high utilization when $C$ is large (e.g., $C \geq 256$ $\mathrm{mb} / \mathrm{s}$ ), the allocated buffer sizes are over provisioned compared to those of ABS. BSCL experiences less loss rate than the BDP and Stanford models, but it still does not lead to a buffer size that satisfies the target loss rate and utilization constraints. ABS again demonstrates the best performance among all these methods, maintaining buffer size within the target performance constraints for all link capacities.

\section{Response to Load Changes}

The volume of traffic perceived by any Internet router is not constant, but exhibits burstiness at different time-scales due to various reasons such as users' demand, route changes, and load balancing. Thus, stability and responsiveness in the presence of load changes is crucial for any buffer sizing scheme purported to operate in practical routers. Hence, we next examine ABS in such a scenario. We still use a "dumbbell" topology where a single bottleneck link of capacity $10 \mathrm{mb} / \mathrm{s}$ is shared by 60 heterogeneous TCP flows. The target utilization $u^{*}=90 \%$ and loss rate $p^{*}=2 \%$. As shown in Figure 4, after all flows start simultaneously at the beginning, both $b(n)$ and $p(n)$ are quickly brought to a close neighborhood of their respective stationary value. At time 48 seconds, 20 flows depart from the system. As a consequence of the reduced traffic load, packet loss rate $p(n)$ immediately drops to $1.1 \%$, which allows the router to release memory space to meet $p^{*}$ in this new scenario. After another 48 seconds, 20 more flows left and again ABS 


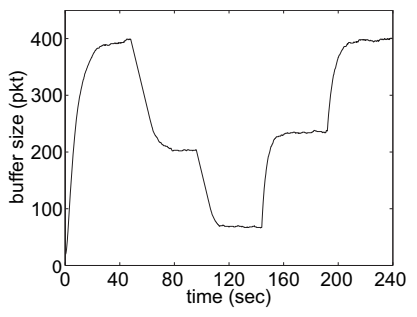

(a) buffer size

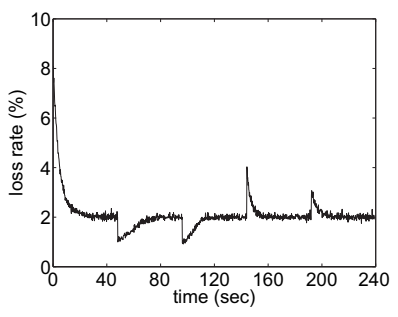

(b) loss rate
Fig. 4. ABS under changing traffic loads $\left(u^{*}=90 \%\right.$ and $\left.p^{*}=2 \%\right)$.

quickly shrinks the buffer. At time 144 and 192 seconds, these two sets of departed flows respectively rejoin the system and $\mathrm{ABS}$ is forced to increase the buffer size. It can be observed from the plots that during the entire simulation, $b(n)$ demonstrates quick responses to load changes, experiences small oscillations in both the transient and steady states, and exhibits smooth transitions between neighboring states.

\section{Web Traffic}

All scenarios considered so far have only long-lived TCP flows. However, the real Internet traffic is composed of a mixture of connections with a wide range of transfer lengths, packet sizes, and RTTs [14]. Thus, to obtain a better understanding of ABS, we next test it in more diverse scenarios.

Consider a network with a single link of capacity 10 $\mathrm{mb} / \mathrm{s}$ shared by 20 persistent FTP flows in the presence of background web traffic generated by 100000 HTTP sessions. Each HTTP session downloads $n_{p}$ pages with inter-page time $t_{p}$ seconds, where $n_{p}$ is uniformly distributed in [10,2000] and $t_{p}$ is exponentially distributed with mean 1 second. Each page contains $n_{o}$ objects where $n_{o}$ is uniformly distributed in $[1,5]$. The inter-object time $t_{o}$ is exponentially distributed with mean 0.01 seconds. Sizes of objects follow the Pareto distribution with mean $\mu=10 \mathrm{~KB}$ and shape parameter $\alpha=1.2$. We set the target utilization $u^{*}=95 \%$ and loss rate $p^{*}=1 \%$.

The simulation results are shown in Figure 5. As observed from Figure 5(a), ABS's behavior in this scenario differs from that of previous simulations in that the buffer size does not converge to a particular value, but fluctuates between 120 and 210 packets due to bursty ingress traffic. Note that this phenomenon does not indicate that ABS is incapable of effectively controlling short-lived web-like traffic, but actually demonstrates that this mechanism is adaptive and responsive in highly dynamic scenarios. This can be clearly seen from Figure 5(b), where utilization $p(n)$ is maintained within a close neighborhood of its target value $p^{*}=95 \%$.

\section{E. Mixture of TCP and Non-TCP Traffic}

Recall that analysis of real Internet traffic traces has demonstrated that although TCP is the predominant transport protocol, a non-eligible portion of Internet traffic is contributed by non-TCP protocols [15]. Thus, in this subsection we examine $\mathrm{ABS}$ in a more diverse environment with $20 \%$ UDP background traffic. Consider a scenario where 20 FTP, 20

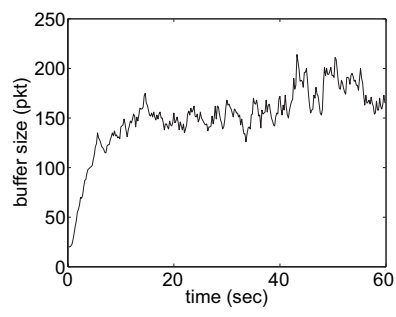

(a) buffer size

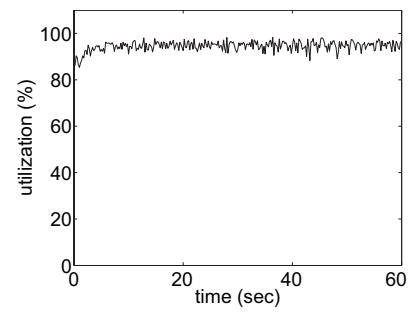

(b) utilization
Fig. 5. ABS in a single link of capacity $10 \mathrm{mb} / \mathrm{s}$ shared by $20 \mathrm{FTP}$ and 100000 HTTP flows $\left(u^{*}=95 \%\right.$ and $\left.p^{*}=1 \%\right)$.

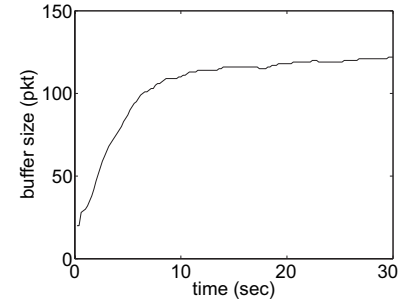

(a) buffer size

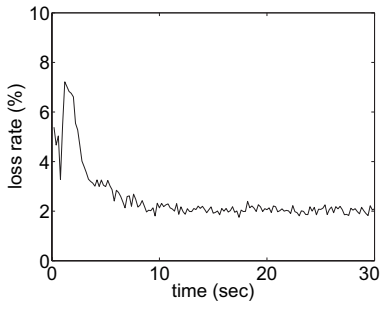

(b) loss rate
Fig. 6. ABS in a single link of capacity $10 \mathrm{mb} / \mathrm{s}$ shared by $20 \mathrm{FTP}, 20$ HTTP, and 20 UDP flows $\left(u^{*}=90 \%\right.$ and $p^{*}=2 \%$ ).

HTTP, and 20 UDP flows compete for resources of a single link of capacity $10 \mathrm{mb} / \mathrm{s}$. Traffic parameters of HTTP flows are the same as the last subsection and each UDP flow transmits packets at a constant rate $0.1 \mathrm{mb} / \mathrm{s}$. We set reference values of the bottleneck router to be $u^{*}=90 \%$ and $p^{*}=2 \%$. The simulation result is shown in Figure 6, where ABS is dominated by the loss constraint and $p(n)$ quickly reaches and subsequently remains in a close neighborhood of $p^{*}$.

According to Section II, the monotonic effects of buffer size $b(n)$ on loss rate $p(n)$ and utilization $u(n)$ should also hold for traffic generated by a set of different congestion control protocols. Thus, we next test ABS under a mixture of TCP variants. Specifically, we preserve values of $p^{*}$ and $u^{*}$, increase the link capacity to $100 \mathrm{mb} / \mathrm{s}$, and synthesize the ingress traffic with 10 Reno, 10 HSTCP, 10 STCP, 10 HTCP, and 10 Westwood flows with RTTs uniformly distributed within $[40,60] \mathrm{ms}$. As illustrated in Figure 7, ABS successfully maintains $u(n)$ around its target value $u^{*}$.

Thus, examples provided in this and the preceding subsections clearly demonstrate ABS's excellent capability of regulating the buffer size under different traffic patterns and transport protocols, making the concept of an ABS-like dynamic scheme a highly versatile and appealing buffer sizing mechanism for real Internet routers.

\section{F. Multi-Link Topology}

We next extend out study to multi-link networks and see whether interactions between multiple ABS routers can produce undesirable effects. Towards this end, consider a two-link "parking lot" topology with three sets of flows. Each set is composed of 20 FTP, 10 HTTP, and 10 UDP (with constant rate $0.1 \mathrm{mb} / \mathrm{s}$ ) flows. These three sets of flows respectively pass 


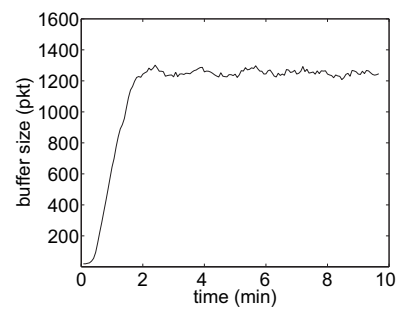

(a) buffer size

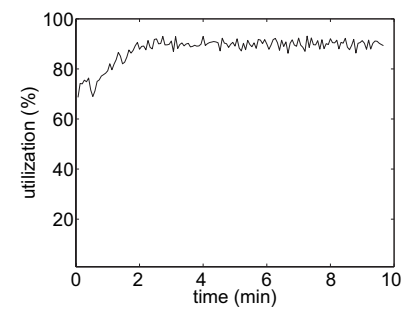

(b) loss rate
Fig. 7. ABS in a single link of capacity $100 \mathrm{mb} / \mathrm{s}$ shared by 10 Reno, 10 HSTCP, 10 STCP, 10 HTCP, and 10 Westwood flows $\left(u^{*}=90 \%\right.$ and $p^{*}=2 \%$ ).

through the first link, second link, and both links. Capacities of these two links are respectively 50 and $20 \mathrm{mb} / \mathrm{s}$. Constraint values are $u_{1}^{*}=95 \%$ and $p_{1}^{*}=1 \%$ for the first link and $u_{2}^{*}=75 \%$ and $p_{2}^{*}=5 \%$ for the second link. As seen from Figure 8, two ABS routers do not intervene each other and successfully maintain utilization at their respective target level.

Based on simulations conducted in this subsection, we have demonstrated that ABS achieves its design goal - regulating buffer size $b(n)$ to satisfy the pre-specified performance constraints. Furthermore, ABS is shown to be stable in the presence of dynamically changing loads and robust to a diverse mixture of long and short TCP flows and even non-TCP traffic. All of these properties make ABS a highly appealing buffer sizing scheme for real Internet routers.

\section{RELATED WORK}

It is commonly suggested that the buffer size $b$ of a bottleneck router should be at least the product of the output link's capacity $C$ and the average round-trip time $R$ of all incoming TCP sessions, i.e., $b \geq C R$. This rule-of-thumb is commonly attributed to Villamizar and Song [34] and is deployed in most current large commercial routers [3]. However, the huge amount of memory space required by this rule becomes progressively unrealistic as link speed of the Internet evolves to the magnitude of multiple giga-bps and even tera-bps.

As pointed out by Appenseller et al. [3], this classic principle is applicable in scenarios where only synchronized longlived TCP flows are present. However, Internet core routers are usually utilized by hundreds of thousands of heterogeneous flows, in which case synchronization rarely happens and the aggregate window size process converges to a Gaussian process [3]. Based on this result, they prove that when router buffers are sized according to $b=C R / \sqrt{N}$, link utilization is lower bounded by $98.99 \%$. This result deviates from the ruleof-thumb in that their suggested buffer sizes scale inversely to the number of flows, indicating that all current backbone routers are over-buffered and their memory space and costs can be substantially reduced.

The small-buffer criteria are extended by Enachescu et al. [10], who suggest that buffers be as small as $10-20$ packets in core routers provided the packet arrival process follows a Poisson distribution. This assumption is enforced

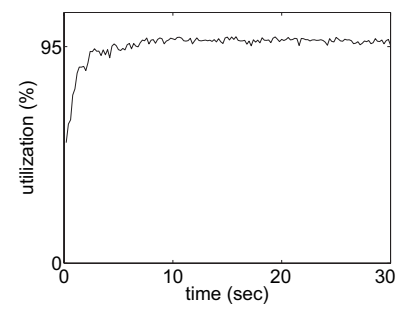

(a) link one

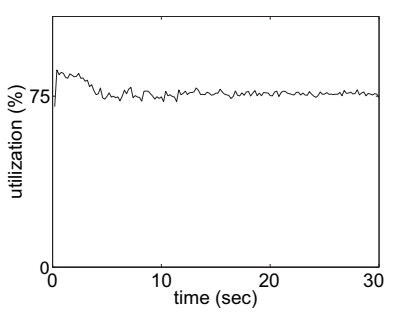

(b) link two
Fig. 8. ABS in a "parking lot" topology $\left(p_{1}^{*}=95 \%\right.$ and $\left.p_{2}^{*}=75 \%\right)$.

by introducing Paced TCP [10], where senders evenly spread out-going packets over an RTT. This result is further extended to the model of combined input-output queue [6] and later supported in [29], [37].

Although the assumption of totally asynchronous flows and Poisson arrivals are sound for backbone routers, as pointed out in [9], generic Internet routers are usually accessed by partially synchronized flows. In this case, the minimum buffer requirement is shown to be [9]:

$$
b=\frac{p(N) C R_{e}-2 S N(1-p(N))}{2-p(N)},
$$

where $R_{e}$ is the harmonic mean of the RTTs, $S$ is the MTU, $p(N)=1-(1-1 / N)^{L_{N}}$ is the fraction of flows that see at least one packet loss, and $L_{N}$ is the average number of dropped packets during a congestion event.

Besides saturating a given link, Dhamdhere et al. [9] propose that minimizing packet loss rate should also be taken into account when sizing router buffers. To accomplish this goal, they develop a buffer management rule based on Flow Proportional Queuing (FPQ), in which the loss rate is kept within a threshold value $p$ by increasing the RTTs (or the buffer size) of the flows proportionally to $N$. Letting $R_{p}$ and $R_{q}^{*}$ respectively be the propagation and required queuing delays of the link, the proposed buffer sizing equation is

$$
b=C R_{q}^{*}=K_{p}^{*} N-C R_{p},
$$

where $K_{p}^{*}=0.87 / \sqrt{p^{*}}$ and $p^{*}$ is the target loss rate. If we consider both utilization and loss constraints, buffer size should be the larger of (17) and (18) and the resulting mechanism is called Buffer Sizing for Congested Link (BSCL) [9]. Note that buffer sizing rule (18) suggests that the bottleneck buffer should linearly increase with $N$, which is in sharp contrast to the aforementioned small-buffer criteria.

Compared to the above schemes that seek to derive an explicit model of buffer size and Internet traffic, another class of methods tries to solve this problem by utilizing a certain implicit relationship between them. Specifically, Shorten et al. [31] propose a method called Adaptive AIMD, which is shown to adapt to any buffer size in the path. In addition, Kellett et al. [23] formulate the relationship between buffer size and utilization as a sector-bounded nonlinearity and develop an adaptive buffer sizing scheme called Adaptive Drop Tail (ADT), whose control equation is given by $b(n)=b(n-1)+K\left(u^{*}-u(n)\right.$, 
where unspecified parameter $K$ needs to satisfy $K \in\left(0,2 / k_{2}\right)$ to achieve stability and $k_{2}$ is the sector nonlinearity upper bound. However, it is unclear how $k_{2}$ is obtained for a given traffic condition and how it can be calculated in real time as the system's dynamics change. Clearly, ADT has to resolve these issues before being used in real Internet routers.

\section{CONCLUSION}

In this paper, we designed and implemented a dynamic buffer sizing scheme, called ABS, that stabilizes the buffer size to its minimum value satisfying given utilization and/or loss constraints. $\mathrm{ABS}$ is composed of two Integral controllers $\mathrm{ABS}_{u}$ and $\mathrm{ABS}_{p}$, each of which is equipped with a parameter training component using a gradient-based technique to achieve the optimal control gains. Besides stability and optimality, an appealing feature of ABS is its robustness to generic Internet traffic composed of long, short, and non-TCP flows. Thus, ABS can significantly benefit router manufactures and ISPs by improving their routers' performance, reducing system cost, and providing QoS guarantees.

We finally note that the emphasis of the paper is not demonstrating superiority of a particular controller, but advocating a new buffer management methodology and presenting the possibility of optimally sizing router buffers using a simple yet robust controller without comprehensive knowledge of Internet dynamics. This controller actually may be replaced by more advanced candidates (e.g., nonlinear PID and variable structure control). Our future work involves designing simpler ABS-like mechanisms, analyzing ABS in its transient phase, studying its stability in more complicated congestion control models, and implementing and testing it in hardware routers under real Internet traffic.

\section{REFERENCES}

[1] M. Allman, V. Paxson, and W. Stevens, "TCP Congestion Control," IETF RFC 2581, Apr. 1999.

[2] H. C. Andersen, A. Lotfi, and A. C. Tsoi, "A New Approach to Adaptive Fuzzy Control: The Controller Output Error Method," IEEE Trans. Syst., Man, Cybern., vol. 27, no. 4, pp. 686-691, Aug. 1997.

[3] G. Appenseller, I. Keslassy, and N. McKeown, "Sizing Router Buffers," in Proc. ACM SIGCOMM, Aug. 2004, pp. 281-292.

[4] S. Athuraliya, S. H. Low, V. H. Li, and Q. Yin, "REM: Active Queue Management," IEEE Networks, vol. 15, no. 3, pp. 48-53, Jun. 2001.

[5] K. Avrachenkov, U. Ayesta, and A. Piunovskiy, "Optimal Choice of the Buffer Size in the Internet Routers," in Proc. IEEE CDC, Dec. 2005, pp. 1143-1148.

[6] N. Beheshti, Y. Ganjali, R. Rajaduray, D. Blumenthal, and N. McKeown, "Buffer Sizing in All-Optical Packet Switches," in Proc. OFC/NFOEC, Mar. 2006.

[7] L. Brakmo, S. O’Malley, and L. Peterson, "TCP Vegas: New Techniques for Congestion Detection and Avoidance," in Proc. ACM SIGCOMM, Aug. 1994, pp. 24-35.

[8] Cisco 3600 Series Routers. [Online]. Available: http://www.cisco.com/ en/US/products/hw/routers/ps274/.

[9] A. Dhamdhere, H. Jiang, and C. Dovrolis, "Buffer Sizing for Congested Internet Links," in Proc. IEEE INFOCOM, Mar. 2005, pp. 1072-1083.

[10] M. Enachescu, Y. Ganjali, A. Goel, N. McKeown, and T. Roughgarden, "Routers with Very Small Buffers," in Proc. IEEE INFOCOM, Apr. 2006.

[11] S. Floyd, "High-speed TCP for Large Congestion Windows," IETF RFC 3649, Dec. 2003.

[12] S. Floyd and T. Henderson, "The NewReno Modification to TCP's Fast Recovery Algorithm," IETF RFC 2582, Apr. 1999.
[13] S. Floyd and V. Jacobson, "Random Early Detection Gateways for Congestion Avoidance," IEEE/ACM Trans. Netw., vol. 1, no. 4, pp. 397413, Aug. 1993.

[14] S. Floyd and E. Kohler, "Internet Research Needs Better Models," ACM SIGCOMM Comp. Comm. Rev., vol. 33, no. 1, pp. 29-34, Jan. 2003.

[15] M. Fomenkov, K. Keys, D. Moore, and K. Claffy, "Longitudinal Study of Internet Traffic from 1998-2003," in Proc. WISICT, Jan. 2004, pp. $1-6$.

[16] Y. Ganjali and N. McKeown, "Update on Buffer Sizing in Internet Routers," ACM SIGCOMM Comp. Comm. Rev., vol. 36, no. 5, pp. 6770, Oct. 2006

[17] M. Gerla, M. Y. Sanadidi, R. Wang, A. Zanella, C. Casetti, and S. Mascolo, "TCP Westwood: Congestion Window Control Using Bandwidth Estimation," in Proc. IEEE GLOBECOM, Nov. 2001, pp. 1698-1702.

[18] S. Gorinsky, A. Kantawala, and J. Turner, "Link Buffer Sizing: A New Look at the Old Problem," in Proc. IEEE ISCC, Jun. 2005, pp. 507-514.

[19] S. Gorinsky, A. Kantawala, and J. Turner, "Simulation Perspectives on Link Buffer Sizing," Simulation, vol. 83, no. 3, pp. 245-257, 2007.

[20] C. V. Hollot, V. Misra, D. Towsley, and W.-B. Gong, "On Designing Improved Controllers for AQM Routers Supporting TCP Flows," in Proc. IEEE INFOCOM, Apr. 2001, pp. 1726-1734.

[21] F. Ishizaki and T. Takine, "Loss Probability in a Finite Discrete-Time Queue in Terms of the Steady State Distribution of an Infinite Queue," Queueing Systems: Theory and Applications, vol. 31, no. 3-4, pp. 317326, Mar. 1999.

[22] C. Jin, D. Wei, and S. H. Low, "FAST TCP: Motivation, Architecture, Algorithms, Performance," in Proc. IEEE INFOCOM, Mar. 2004, pp. 2490-2501.

[23] C. Kellett, R. Shorten, and D. J. Leith, "Sizing Internet Router Buffers, Active Queue Management, and the Lur'e Problem," in Proc. IEEE CDC, Dec. 2006, pp. 650-654

[24] T. Kelly, "Scalable TCP: Improving Performance in High-speed Wide Area Networks," Computer Communication Review, vol. 33, no. 2, pp. 83-91, Apr. 2003.

[25] A. Kuzmanovic and E. Knightly, "TCP-LP: a Distributed Algorithm for Low Priority Data Transfer," in Proc. IEEE INFOCOM, Apr. 2003, pp. 1691-1701.

[26] D. Leith and R. Shorten, "H-TCP Protocol for High-Speed Long Distance Networks," in Proc. PFLDnet, Feb. 2004.

[27] S. H. Low, F. Paganini, J. Wang, S. Adlakha, and J. Doyle, "Dynamics of TCP/RED and a Scalable Control," in Proc. IEEE INFOCOM, Jun. 2002, pp. 239-248.

[28] R. Prasad, C. Dovrolis, and M. Thottan, "Router Buffer Sizing Revisited: The Role of the Output/Input Capacity Ratio," in Proc. ACM SIGCOMM CoNEXT, Dec. 2007

[29] G. Raina, D. Towsley, and D. Wischik, "Part II: Control Theory for Buffer Sizing," ACM SIGCOMM Comp. Comm. Rev., vol. 35, no. 3, pp. 79-82, Jul. 2005

[30] I. Rhee and L. Xu, "CUBIC: A New TCP-Friendly High-Speed TCP Variant," in Proc. PFLDnet, Feb. 2005.

[31] R. N. Shorten and D. J. Leith, "On Queue Provisioning, Network Efficiency and the Transmission Control Protocol," Hamilton Institute, Tech. Rep., 2006.

[32] J. A. Snyman, Practical Mathematical Optimization: An Introduction to Basic Optimization Theory and Classical and New Gradient-Based Algorithms. Springer, 2005.

[33] J. Sommers, P. Barford, A. Greenberg, and W. Willinger, "An SLA Perspective on the Router Buffer Sizing Problem," ACM SIGMETRICS Performance Evaluation Review, vol. 35, no. 4, Mar. 2008.

[34] C. Villamizar and C. Song, "High Performance TCP in the ANSNET," ACM SIGCOMM Comp. Comm. Rev., vol. 24, no. 5, pp. 45-60, Oct. 1994.

[35] D. X. Wei and P. Cao, "NS-2 TCP-Linux: An NS-2 TCP implementation with congestion control algorithms from Linux," in Proc. WNS2, Oct. 2006.

[36] D. Wischik, "Buffer Requirements for High-Speed Routers," in Proc. ECOC, Sep. 2005, pp. 23-26.

[37] D. Wischik and N. McKeown, "Part I: Buffer Sizes for Core Routers," ACM SIGCOMM Comp. Comm. Rev., vol. 35, no. 2, pp. 75-78, Jul. 2005.

[38] L. Xu, K. Harfoush, and I. Rhee, "Binary Increase Congestion Control (BIC) for Fast, Long Distance Networks," in Proc. IEEE INFOCOM, Mar. 2004, pp. 2514-2524. 